\title{
Skyrmions in magnetic multilayers: chirality, electrical detection and current-induced motion
}

\author{
Nicolas Reyren $^{\mathrm{a}}$, Karim Bouzehouane ${ }^{\mathrm{a}}$, Jean-Yves Chauleau ${ }^{\mathrm{a}, \mathrm{b}}$, Sophie Collin ${ }^{\mathrm{a}}$, Albert Fert ${ }^{\mathrm{a}}$, \\ Simone Finizio $^{c}$, Karin Garcia ${ }^{\mathrm{a}}$, Sean Hughes ${ }^{\mathrm{d}}$, Nicolas Jaouen ${ }^{\mathrm{b}}$, William Legrand ${ }^{\mathrm{a}}$, Davide \\ Maccariello $^{\mathrm{a}}$, Samuel McFadzean ${ }^{\mathrm{d}}$, Stephen McVitie ${ }^{\mathrm{d}}$, Christoforos Moutafis ${ }^{\mathrm{e}}$, Horia Popescu ${ }^{\mathrm{b}}$, \\ Jörg Raabe ${ }^{\mathrm{c}}$, C. A. F. Vaz ${ }^{\mathrm{c}}$, and Vincent Cros $^{\mathrm{a}}$ \\ aUnité Mixte de Physique, CNRS, Thales, Univ. Paris-Sud, Université Paris-Saclay, Palaiseau, \\ France \\ bynchrotron SOLEIL, L'Orme des Merisiers, 91192, Gif-sur-Yvette, France \\ ${ }^{\mathrm{c} S w i s s}$ Light Source, Paul Scherrer Institut, 5232 Villigen, Switzerland. \\ ${ }^{\mathrm{d}}$ Scottish Universities Physics Alliance, School of Physics and Astronomy, University of \\ Glasgow, Glasgow G12 8QQ United Kingdom

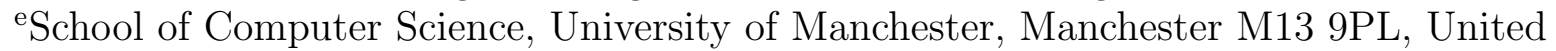 \\ Kingdom
}

\begin{abstract}
Sub-100-nm skyrmions are stabilized in magnetic metallic multilayers and observed using transmission electron microscopy, magnetic force microscopy, scanning transmission X-ray microscopy and X-ray resonant magnetic scattering. All these advanced imaging techniques demonstrate the presence of "pure" Néel skyrmion textures with a determined chirality. Combining these observations with electrical measurements allows us to demonstrate reproducible skyrmion nucleation using current pulses, and measure their contribution to the transverse resistivity to detect them electrically. Once nucleated, skyrmions can be moved using charge currents. We find predominantly a creep-like regime, characterized by disordered skyrmion motion, as observed by atomic force microscopy and scanning transmission X-ray microscopy. These observations are explained qualitatively and to some extent quantitatively by the presence of crystalline grains of about $20 \mathrm{~nm}$ lateral size with a distribution of magnetic properties.
\end{abstract}

Keywords: skyrmion, nucleation, XRMS, STXM, MFM, Néel, domain wall, spin torque, metal, multilayer

\section{INTRODUCTION}

Magnetic skyrmions are two-dimensional (2D) whirling magnetization textures with a specific topological number $N_{\mathrm{sk}}= \pm 1$, distinct from the uniform ferromagnetic state $N_{\mathrm{sk}}=0$, with $N_{\mathrm{sk}}=1 /(4 \pi) \int_{S} \vec{m} \cdot\left(\partial_{x} \vec{m} \times \partial_{y} \vec{m}\right) \mathrm{d} x \mathrm{~d} y$, $S$ being the surface of the magnetic texture with local normalized magnetization $\vec{m} .{ }^{1}$ This topological number proper to skyrmions can also been found in magnetic bubbles, but unlike bubbles stabilized by the dipolar interaction and perpendicular magnetic anisotropy only, skyrmions have additionally a fixed chirality and $N_{\text {sk }}$. More stringent definitions of a skyrmion require a point-like core, an aspect that is difficult to probe experimentally, except perhaps with spin-polarized scanning transmission microscopy. ${ }^{2,3}$ Often a chiral exchange interaction is needed in order to stabilize the skyrmion, which can be provided by the Dzyaloshinskii-Moriya (DM) interaction, the antisymmetric analog of the Heisenberg exchange interaction, with Hamiltonian $H=\vec{D}_{i j} \cdot\left(\vec{S}_{i} \times \vec{S}_{j}\right)$ between spins $S_{i}$ and $S_{j}$, with $\vec{D}_{i j}$ the DM vector. The DM interaction (DMI) requires spin-orbit coupling and inversion symmetry breaking, the latter being provided by the crystalline structure (e.g. B20) or by interfaces. Interfaces are interesting because they provide possibilities to engineer DMI, varying the materials in contact to the ferromagnetic layer, varying thicknesses, coupling different materials, etc. In our sputtered multilayer films,

Further author information: (Send correspondence to N.R.)

N.R.: E-mail: nicolas.reyren@cnrs-thales.fr, Telephone: +33169415839

Spintronics X, edited by Henri-Jean Drouhin, Jean-Eric Wegrowe, Manijeh Razeghi, Henri Jaffrès, Proc. of SPIE Vol. 10357, 1035724 - (c) 2017 SPIE · CCC code: 0277-786X/17/\$18 · doi: 10.1117/12.2275058 
we observe interfacial DM interaction with $\vec{D}_{i j}$ pointing in the plane of the films, in a direction orthogonal to the vector pointing from $S_{i}$ to $S_{j}$. The energy related to such DM interaction is hence minimized for cycloidal spin textures, rather than helical, corresponding respectively to the Néel and Bloch domain wall (DW) textures of specific chirality.

After the first reports demonstrating the presence of skyrmions in single atomic layers at low temperatures (see reviews 4,5), several ways of stabilizing these magnetic structures at room temperature (RT) were explored. We concentrate on metallic systems, which seem more relevant for applications. One solution to stabilize a magnetic texture against thermal fluctuations is to increasing the magnetic volume, but in order to keep a strong enough interfacial DMI and interface perpendicular magnetic anisotropy (PMA), the ferromagnetic layer cannot be increased at will. Hence, we grew multilayers coupled by dipolar fields, with each ferromagnetic interface engineered to provide PMA and DMI (Fig.1(a)). We concentrated on the Co|Pt interface for which the DMI magnitude can reach about $2 \mathrm{~mJ} / \mathrm{m}^{2}$ (see e.g. 6). However, to avoid the canceling of the DMI interaction in symmetric structures, a third layer needs to be introduced, such as $\mathrm{Ir}, \mathrm{Ta}$, or $\mathrm{Al}_{2} \mathrm{O}_{3}$. Indeed, if Co has interfaces with $\mathrm{Pt}$ on the top and bottom interfaces, the effective DMI is close to zero, because the DMI emerging at both interfaces have opposite DM vector (Fig.1(c-d)).7,8

\section{SAMPLES: DESIGN, GROWTH AND CHARACTERIZATION}

We deposit our films at room temperature by magnetron sputtering on thermally oxidized $\mathrm{SiO}_{2} \mathrm{substrates}_{\text {for }}$ most experiments and on X-ray transparent $\mathrm{Si}_{3} \mathrm{~N}_{4}$ membranes for transmission experiments, namely, scanning transmission X-ray microscopy (STXM) and transmission electron microscopy (TEM). TEM cross-sections of multilayers indicates continuity of the Co layers on large scales (Fig.1(b)). This is further confirmed by X-ray low angle reflectivity (Fig.2), which also indicates that the film thicknesses are close to the nominal thicknesses and that film roughness propagates coherently.

\section{ADVANCED IMAGING TOOLS: REVEALING THE MAGNETIC TEXTURE}

\subsection{Lorentz-mode transmission electron microscopy}

TEM in Lorentz mode has been used in order to verify the Néel character of the DW made of Pt|Co|Ir samples: no contrast appears on images at normal incidence, while rotating the sample reveals paired dark and bright arcs aligned along the rotation axis. ${ }^{9}$ (Skyrmions were stabilized using the field of the electron beam microscope lenses.) Signal from the center of the DW has not yet been detected, leaving the chirality of the DW undetermined between either clockwise $(\mathrm{CW})$ or counter-clockwise $(\mathrm{CCW})$ Néel. However, the estimated DMI magnitude in this system implies that the chirality must be fixed and that only one type of Néel domain wall should be present.

\subsection{Scanning transmission X-ray microscopy}

In similar samples, we performed STXM imaging at the Co $L_{3}$ edge at about $778 \mathrm{eV}{ }^{6}$ STXM technique has the advantage of rather directly measuring the local out-of-plane magnetization $m_{z}$ when circularly polarized light is used in normal incidence: more precisely $m_{z} \propto \mu_{\mathrm{CL}}-\mu_{\mathrm{CR}}$ where $\mu_{*}$ are the absorption coefficient for circular left (CL) and circular right (CR) polarization of the photons. Measurements were performed at the PolLux beamline at the Swiss Light Source synchrotron (PSI, Switzerland) with a setup that allows one to apply an out of plane field during the STXM measurement. The magnetization profile can be compared to simulated profiles taking into account the beam shape, ${ }^{6}$ or alternatively, can be deconvoluted assuming again a particular beam shape. ${ }^{10}$ Such a deconvoluted profile is plotted in Fig.3(b). We also checked that the skyrmions have similar sizes, with a very weak field dependence above about $50 \mathrm{mT}$ (depending on the sample). Once skyrmions are formed, their sizes (defined by the diameter $d_{\mathrm{sk}}$ of the circle with $m_{z}=0$ ) do not vary significantly and exhibit no sizable magnetic field hysteresis (Fig.3(c)). As we demonstrated before using micromagnetic simulations, ${ }^{6}$ such sub-100-nm skyrmions are not stabilized by dipolar coupling only and a finite DMI magnitude is required to stabilize them. From this study, it was already clear that the skyrmions must have a fixed chirality due to the large involved DMI, however the detailed texture could not be determined, due to the limited transverse resolution of the STXM microscope. 

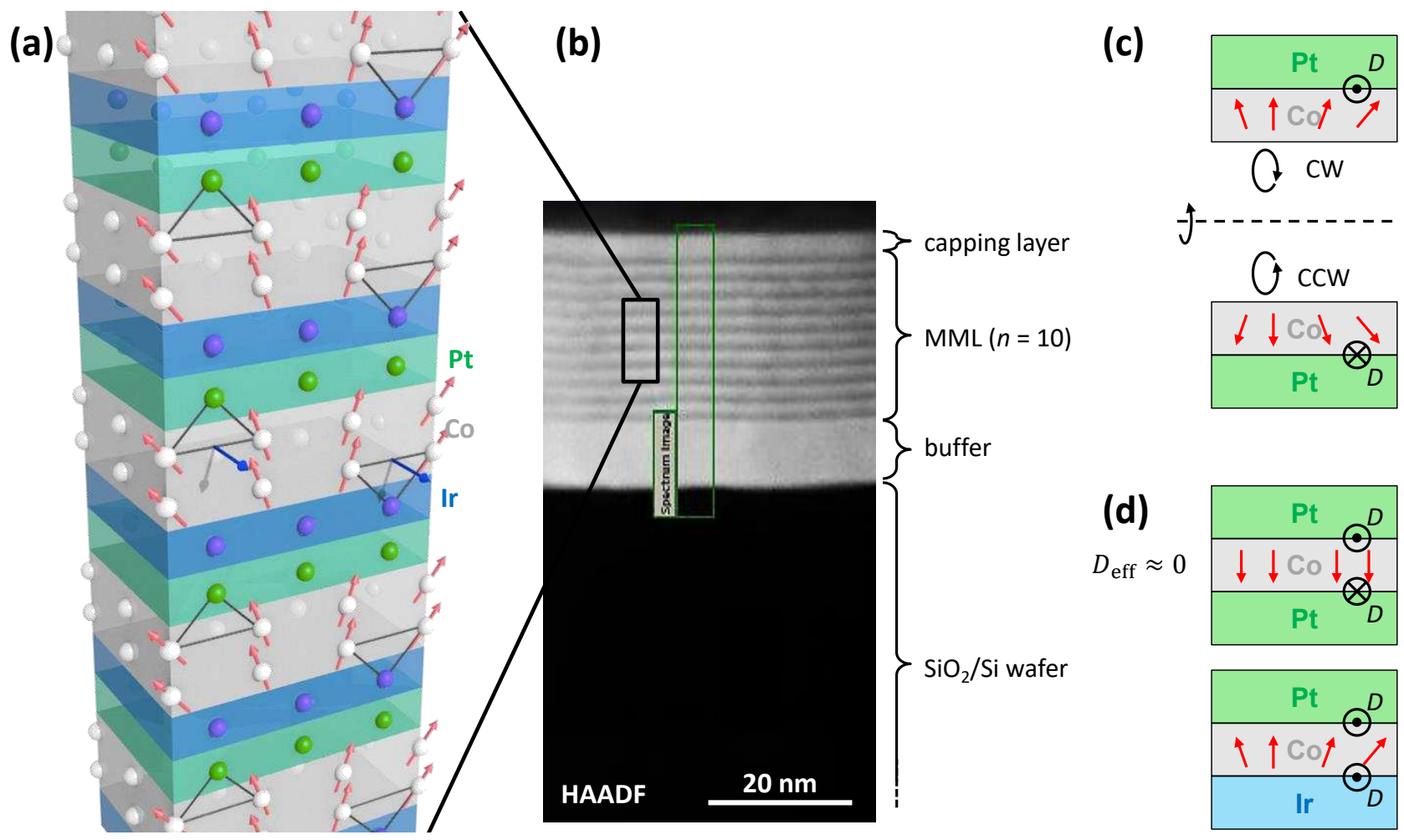

Figure 1. Structure of the studied samples. (a) Artistic representation of the magnetic multilayers made of repetitions of $\mathrm{Pt}|\mathrm{Co}| \mathrm{Ir}$. (b) Actual transmission electron microscopy cross-section displaying continuous Co layers, nominally 0.8-nm thick. (c-d) Illustration of the need to have different top and bottom interface to obtain large effective DMI: (c) by symmetry, rotating the sample around the dashed line, one easily verifies that the DM vector changes sign for the top interface and hence compensates the DM vector of the bottom interface. (d) Choosing a material with opposite $D$, on the contrary, allows one to enhance the DMI.

\subsection{X-ray resonant magnetic scattering}

STXM $^{6}$ and Lorentz-TEM ${ }^{9,11}$ strongly suggest that all skyrmions have a fixed Néel texture. The remaining question was the sign of rotation of the magnetization through the skyrmions (CW or CCW). Recent experiments using X-ray resonant magnetic scattering (Fig.4(a)) at the SEXTANTS beamline (SOLEIL synchrotron, France) allowed us to unambiguously determine the chirality of the DW in our films, ${ }^{12}$ in a similar way that was used for thicker films to detect closure domains. ${ }^{13}$ Using again circularly polarized light with energy set to the Co $L_{3}$ edge, we could observe circular dichroism in the diffracted pattern corresponding to the magnetization of the sample, which were demagnetized to generate labyrinthine domains (Fig.4(b)). The ring obtained by XRMS with unpolarized light has the same diameter as the Fourier transform of the MFM image, confirming that the magnetic domains are indeed at the origin of the diffraction pattern. We found that the pattern of the diffracted ring in circular dichroism directly indicates the DW texture, without any a priori knowledge of the sample: CW and CCW Néel texture give opposite signal, while Bloch textures are rotated by $\pi / 2$ compared to the Néel ones. ${ }^{12}$ Other advantages of this technique include that the magnetic domains are unaffected by the light, samples are still measurable below several $\mathrm{nm}$ of capping layer, insulating samples are imaged as well as conducting ones, no lithography is needed, external magnetic fields are applicable, and measurement times are short (in the range of a few minutes). XRMS could therefore become the method of choice to determine skyrmion chirality, and more generally to measure DW textures. Note however, that in some cases, it also reveals more complexity than one may naively expect, and the corresponding data analysis become more complicated. 


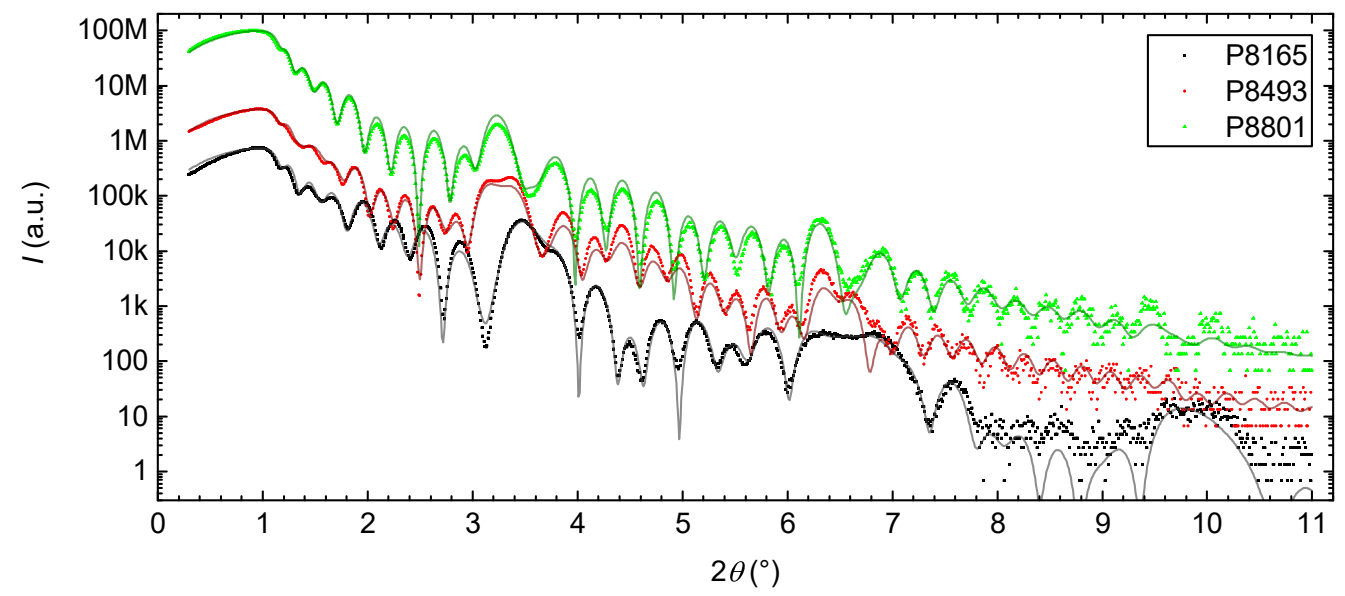

Figure 2. Low angle X-ray reflectivity (using a laboratory $\mathrm{Cu} K_{\alpha}$ source) of a series of five repetitions samples with nominal composition: P8165 ||Pt10|(Ir1|Co0.8|Pt1)x5|Pt3, P8493 ||Ta5|Pt10|(Co0.8|Ir1|Pt1)x5|Pt3, and P8801 ||Pt11|(Co0.8|Ir1|Pt1)x5|Pt3 (numbers after the elements are thicknesses in $\mathrm{nm}$ and $\|$ designates the $\mathrm{SiO}_{2}$ substrate, a.u. stands for arbitrary units, $\theta$ is the X-ray incidence angle). Experimental data are fitted using X'Pert Reflectivity PANalytical software, which confirms the nominal multilayer thicknesses.

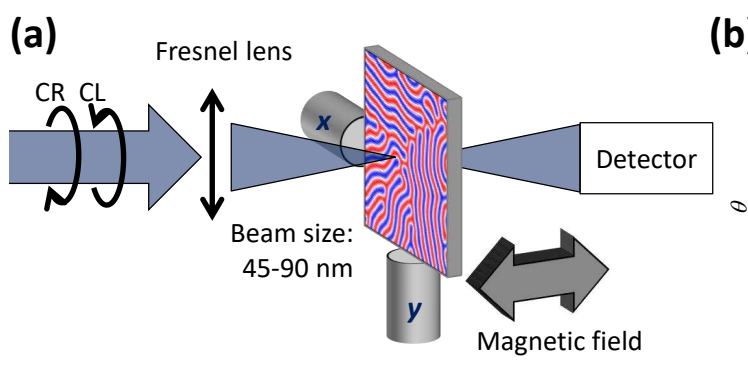

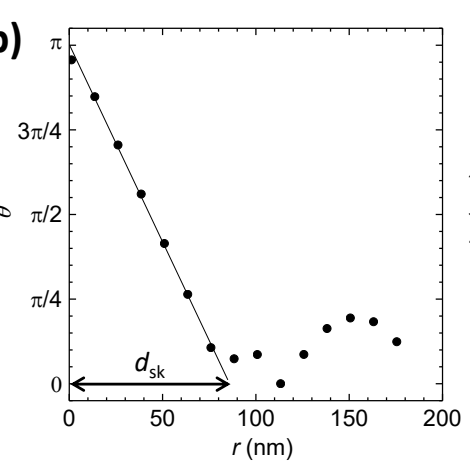

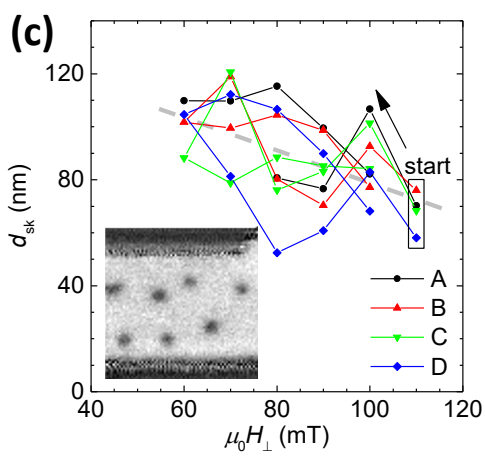

Figure 3. Scanning X-ray transmission microscopy using circularly polarized light. (a) Schematic view of the measurement setup: X-ray at the Co L $\mathrm{L}_{3}$ edge energy is focused on the sample, which is scanned in the beam, and the transmitted photon intensity is measured. An external magnetic field can be applied perpendicular to the sample. (b) Profile of a skyrmion as measured by STXM. The angle $\theta$ with the normal to the film as a function of the skyrmion radius $r$ is deduced from $m_{z}$, which is obtained by deconvoluting the difference in absorption for CL and CR light. (c) Measurement of four skyrmion diameters $d_{\mathrm{sk}}$ as a function of the out of plane magnetic field. Inset: corresponding STXM dichroic image, $1.4 \times 1.4 \mu \mathrm{m}^{2}$.

\section{TRANSPORT MEASUREMENTS: SKYRMION NUCLEATION BY CURRENT PULSES AND ELECTRICAL SIGNATURE OF THE SINGLE SKYRMION}

Having now conclusively demonstrated that the skyrmions stabilized in our magnetic multilayers have all a CW (CCW) Néel structure for films in which Co is below (above) the Pt layer, we can ask ourselves about topological effects in the transport properties. In the following, we will first summarize recent results concerning skyrmions nucleation and their electrical signature in Hall cross geometry. We will then discuss the observed motion of skyrmions using current pulses, discuss their disordered motion and finally discuss more generally the motion of such Néel skyrmions in idealized systems.

\subsection{Combined magnetic force microscopy and electrical transport}

At our exploratory stage, having the possibility to "see" skyrmions at the same time as we excite our samples by external magnetic field and charge current is essential for a better understanding of the physical and material 
(a)

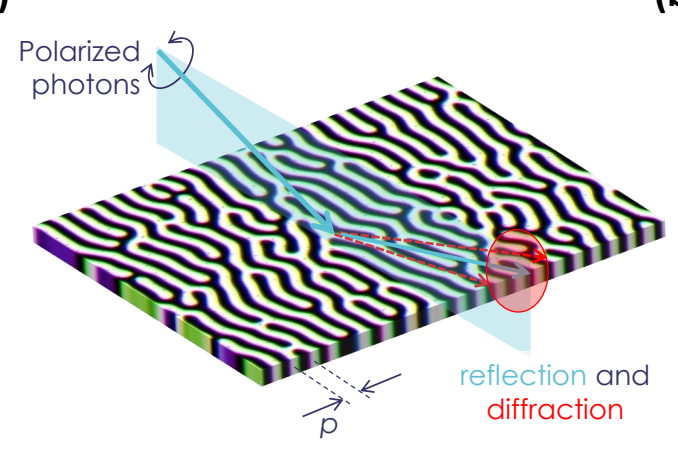

(b)

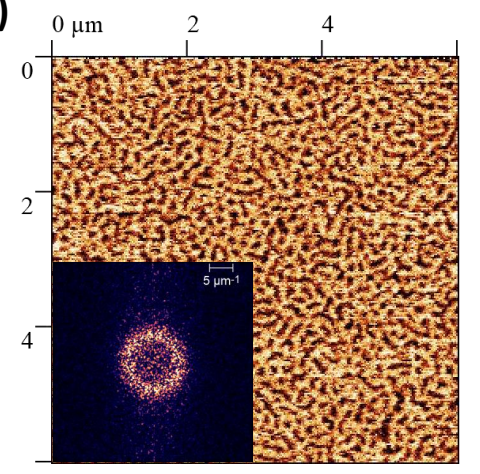

Figure 4. (a) Principle of the X-ray resonant magnetic scattering using circularly polarized light. (b) Magnetic force micrograph (phase map) of a || $\mathrm{Pt} 10|(\operatorname{Ir} 1|\mathrm{Co} 0.8| \mathrm{Pt} 1) \times 5| \mathrm{Pt} 3$ sample with labyrinthine domains. Inset: Fourier transform of the image.

properties of these skyrmion systems. Therefore, we developed a setup built around an Asylum Research MFP$3 \mathrm{D}$ microscope that allows us to connect the sample, as schematically represented in Fig.5. Also the connections were not optimized for fast pulses, they still allow current pulses down to about 50-100 ns to be transmitted through the sample.
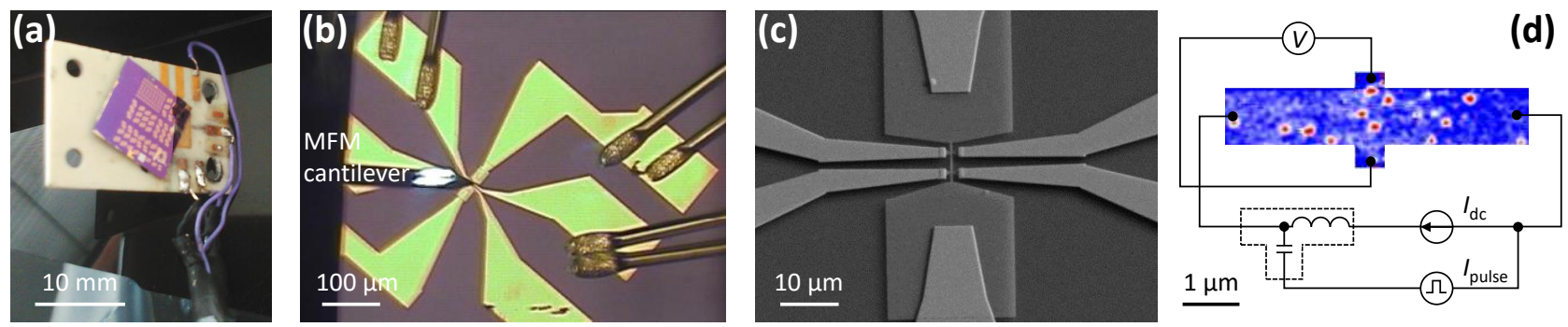

Figure 5. Combined transport-imaging setup. (a) View of the patterned sample mounted on the sample holder with connections to BNC cables. (b) Optical view of a sample in the MFM microscope with wire-bonded connections. (c) Scanning electron micrograph of a patterned sample. (d) Actual MFM image (phase map) of a sample and its schematic wiring.

\subsection{Skyrmion nucleation by current pulses and its physical origin}

While trying to move skyrmions using current pulses of 100 or $200 \mathrm{~ns}$ duration, we discovered ${ }^{14}$ that $^{\text {we could }}$ nucleate skyrmions above a certain current density threshold, as it was already reported in micro-structures with a particular geometry ${ }^{15}$ and as was suggested by numerical simulations ${ }^{16,17}$ (Fig.6(a-b)). While often a complex nucleation process related to the spin torque in geometrically constricted circuits are invoked, ${ }^{15,16}$ we have strong indications that the nucleation process in our simple straight tracks is related to a thermal process. First, the thermal stability of a magnetic object or texture is proportional to its volume, and we observed indeed in a series of $1-\mu \mathrm{m}$-tracks with variable Co thickness $t_{\mathrm{Co}}|| \mathrm{Ta} 15 \mid$ Co $t_{\mathrm{Co}}\left|\left(\mathrm{Pt} 1|\operatorname{Ir} 1| \mathrm{Co} t_{\mathrm{Co}}\right) \mathrm{x} 10\right| \mathrm{Pt} 3$, that the injected Joule heating energy $\propto J_{n}^{2}$ (where $J_{n}$ is the current density) is proportional to the magnetic volume $\propto t_{\text {Co }}$ (Fig.6(c)). Note that by linear extrapolation, one can predict the onset of thermal instabilities at a thickness between 0.4 and $0.5 \mathrm{~nm}$, indicating that room temperature is enough to destabilize small magnetic textures as our skyrmions for $t_{\mathrm{Co}}<0.4 \mathrm{~nm}$. The estimated temperature rise due to the current pulses is still below the Curie temperature, $T_{c}$, but may overcome the magnetic anisotropy (changing sign at $T<T_{c}$ ), effectively erasing the out of plane textures. The second indication of the thermal origin of the skyrmion nucleation lies in the dependence of the threshold voltage $V_{n}$ with the repetition rate or the duty cycle $\eta$. In a reasonable range of $\eta$, 
the injected power $P$ leads to a steady rate of temperature increase, up to a critical point $T_{n}$ where skyrmions are generated: $T_{n}-T_{\mathrm{RT}} \propto P \propto V_{n}^{2} \eta$ and hence $V_{n}^{2} \propto 1 / \eta$ as observed in Fig.6(d). We did not plot $J_{n}$ because of the imperfect impedance matching of the circuit, and the offset corresponding to $a$ in the fit is likely to be related to the final pulse width.

The density of nucleated skyrmions depends on the external magnetic field: the higher the field applied opposite to the skyrmion core, the lower their density. ${ }^{10}$ Now that skyrmions can be nucleated at will with current pulses, we can study their contribution to electrical signals in simple circuits such as the one illustrated in Fig.5(d).
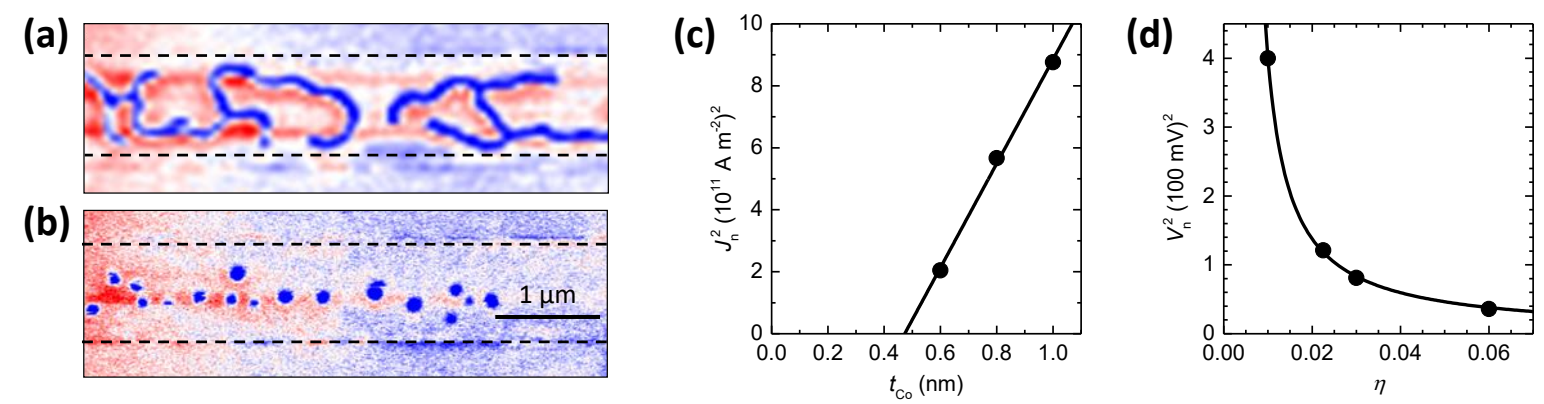

Figure 6. Current-induced skyrmion nucleation. (a) Domain structure as observed by MFM (phase map) after field demagnetization in a 1- $\mu$ m-track made of || Ta15|Co1|(Pt1|Ir1|Co1)x10|Pt3. (b) Same track area after sending one thousand pulses, 200-ns-long and corresponding to a current density $J=0.3 \mathrm{TA} / \mathrm{m}^{2}$. (c) Threshold current density $J_{n}$ squared for skyrmion nucleation as a function of the Co thickness $t_{\mathrm{Co}}$ (proportional to the magnetic volume). The line is a linear fit. (d) Threshold voltage $V_{n}$ squared (proportional to $J_{n}^{2}$ ) as a function of the duty cycle $\eta$. The line is a fit of the form $V_{n}^{2}=1 /(a+b \eta)$, with $a$ and $b$ constants.

\subsection{Electrical skyrmion detection through transverse resistivity measurements}

We nucleated different densities of skyrmions in Hall bar structures such as the one pictured in Fig.5(d), and measured the transverse resistivity $\rho_{x y} .{ }^{10}$ From MFM measurements, we can measure the nulber of skyrmions present in the track and correlate it with a change in $\rho_{x y}$. For our metallic magnetic multilayers with skyrmions diameters of $85 \pm 10 \mathrm{~nm}$, we find a linear relationship, leading to a single skyrmion contribution $\Delta \rho_{x y}=3.5 \pm 0.5 \mathrm{n} \Omega \mathrm{cm}$. In B20 materials, it has been reported that topological Hall effect (THE) has an important contribution to $\rho_{x y} .{ }^{18}$

We evaluated the expected signal for both this THE and the common anomalous Hall effect (AHE), which is, as we experimentally checked in our metallic multilayers, proportional to the mean $m_{z}$ in the Hall bar. The AHE signal related to a single skyrmion is then simply $\Delta \rho_{x y}^{\mathrm{AHE}}=\left.\rho_{\mathrm{AHE}} \bar{m}_{z}\right|_{\mathrm{sk}} \pi d_{\mathrm{sk}}^{2} / a_{\mathrm{cross}}=\rho_{\mathrm{AHE}}\left(16 a_{\mathrm{sk}}\right) /\left(\pi^{2} a_{\text {cross }}\right) \approx$ $3.3 \mathrm{n} \Omega \mathrm{cm}$, where $\rho_{\mathrm{AHE}}=360 \mathrm{n} \Omega \mathrm{cm}$ is the total anomalous Hall effect of the uniform ferromagnetic state, $\left.\bar{m}_{z}\right|_{\mathrm{sk}}=4 / \pi^{2}$ is the mean $m_{z}$ over the skyrmion area considering the experimental profile by STXM (Fig.3(b)), $a_{\text {cross }}$ and $a_{\text {sk }} \equiv \pi d_{\mathrm{sk}}^{2} / 4$ are the Hall cross and skyrmion area. The AHE contribution is hence sufficient to explain the observed $\Delta \rho_{x y}$. An upper bound to the THE can be evaluated by considering that electrons keep their phase through the skyrmions and that each of the latter is responsible for a quantum of emerging flux $\Phi_{0}$. This means that the elastic mean free path should be of the order of $100 \mathrm{~nm}$, which is most probably a large overestimation. If moreover, one considers a 100\% polarization of the conducting electrons (in Co, one expects about 60\%), the naive expression of the THE contribution to the transverse resistivity should be $\Delta \rho_{x y}^{\mathrm{THE}}=\rho_{\mathrm{OHE}} \Phi_{0} / a_{\text {cross }} \approx 4 \cdot 10^{-3} \mathrm{n} \Omega \mathrm{cm}$, with $\rho_{\mathrm{OHE}}=2 \cdot 10^{-11} \Omega \mathrm{m} / \mathrm{T}$ the measured ordinary Hall coefficient.

The THE contribution is hence orders of magnitude weaker than the AHE one, which explains alone the observed signal. ${ }^{10}$ Irrespective of its origin, this signal allows one to count skyrmions in a "large" $1-\mu \mathrm{m}$-wide Hall bar, and gives enough signal to detect single skyrmion in future devices with sizes adapted to accommodate only single rows of skyrmions. ${ }^{10,19}$ 


\section{SKYRMION MOTION UNDER CURRENT PULSES}

\subsection{Observation of disordered motion, "depinning" current and mobility}

For current densities below the nucleation threshold (which also depends of the pulse width), it has been possible to observe by MFM and STXM the motion of the skyrmions. However, we do not observe a uniform velocity. On the contrary, the trajectories of moving skyrmions are meandering and no clear skyrmion Hall effect is observed, ${ }^{14}$ unlike in other systems. ${ }^{20}$ We also observe that some skyrmions do not move at all, some move when pushed by another, and finally, some are annihilated or nucleated.

These observations can be well reproduced qualitatively in micromagnetic simulations, where grains with different properties are considered, e.g. with DMI magnitude varying from one grain to the other. To perform these simulations, we used MuMax $3^{21}$ considering a perpendicular spin current, i.e. in a spin Hall effect configuration. The effect is qualitatively the same for any grain-to-grain varying micromagnetic parameter that we studied: exchange, PMA, DMI and magnetization, or thickness (assuming purely interfacial PMA and DMI). ${ }^{14,22}$ Note that similar results were also obtained for a particle-based model with pinning sites. ${ }^{23}$ We can even find a quantitative match with experiments, but for sets of parameters (magnitude and dispersion of the grain-to-grain magnetic properties) that we cannot verify yet. Below a current threshold, we can observe the same phenomena than by MFM: for example, in the case of DMI variation (Fig.7(a)), skyrmions can be trapped in a grain of large DMI, will move around low DMI grains, sometimes will get stuck at some grain boundaries and then freed by another skyrmion. The resulting average velocity is lower than at single grains, and the skyrmion Hall effect disappears. At large enough currents, the skyrmions recover a "flow" motion with straight trajectories and display a skyrmion Hall effect as expected. Interestingly, even at extremely large currents $\left(>4 \mathrm{TA} / \mathrm{m}^{2}\right)$, they do not recover the same velocity $v$ than skyrmions in homogeneous media, but they regain the same mobility $\mu=\mathrm{d} v / \mathrm{d} J .^{14}$

The transition from a creep-like regime (the process is distinct from that occurring in DW motion) to the flow regime occurs at a current density $J_{0}$, which varies with the grain size. In fact, as displayed in Fig.7(b), the current density required to exit the creep regime is maximal when the grain size matches the skyrmion diameter. The grain sizes observed in our films by normal incidence TEM show a log-normal size distribution centered at about $4 \mathrm{~nm}$, one order of magnitude smaller than the observed skyrmion diameter. However, larger grains corresponding to the skyrmion size exist at the tail of the distribution and might constitute the "pinning centers" observed in MFM.

(a)

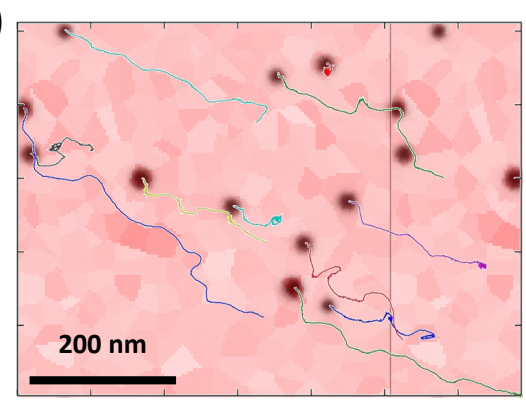

(b)

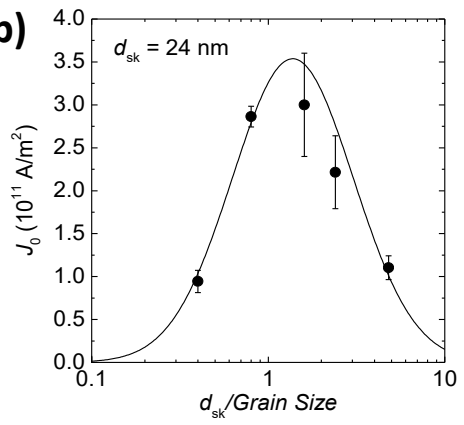

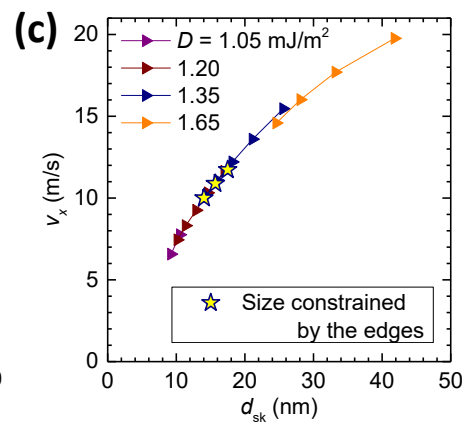

Figure 7. Current-induced skyrmion motion in a system with non-uniform crystalline grain distribution. (a) Image of a simulation landscape (grains of different pink levels are grains with different $D, 10 \%$ variation considered here). The trajectories (colored lines) are calculated using micromagnetic calculations, skyrmions are drawn at their initial position. (b) "Depinnning" current as a function of the ratio between the skyrmion diameter and the grain sizes. (c) Skyrmion velocity as a function of skyrmion diameter at an edge or in a track.

\subsection{Size and velocity: a theoretical and numerical perspective}

What controls the velocity of skyrmions, besides inhomogeneities and defects? We start from the Thiele equation $\vec{G} \times \vec{v}-\alpha \mathbf{D} \vec{v}+\vec{F}=0$, where $\vec{G}$ is the gyrovector, $\alpha$ is the damping, $\mathbf{D}$ is the damping tensor, and $\vec{F}$ is the 
sum of the external forces. We consider here the force related to the damping-like torque generated by the spin Hall effect on a perfectly Néel skyrmion $F \approx J_{s} \pi^{2} d_{\mathrm{sk}} / 2$ in the limit of $d_{\mathrm{sk}} \gg \Delta, \Delta$ being the DW width and $J_{s}$ the injected spin current. In these limits, one finds that the skyrmion Hall angle varies as $4 \Delta /\left(\alpha d_{\mathrm{sk}}\right)$ and the velocity is proportional to $d_{\mathrm{sk}} J_{s}$ to first order of approximation.

We could verify this analytic approach by micromagnetic simulations in which the skyrmion size is modulated by field and DMI magnitude. In Fig.7(c), we trace the velocity as a function of the diameter of the skyrmion in the case where the repulsion force of an edge is also considered. This situation is the one found in tracks. It is found that the skyrmion velocity scales with size, independently of the details of the micromagnetic parameters used in the simulation. Even if the skyrmion size is reduced due to the final size of the track, the same scaling is observed.

The consequence of this observation is that smaller skyrmions are expected to be slower. However, having skyrmion race-track application in mind, this is not an obstacle, as the expected reading rate will scale as $v / d_{\mathrm{sk}} \approx$ constant, $d_{\mathrm{sk}}$ giving the linear density of information.

\section{CONCLUSIONS AND PERSPECTIVES}

The quest for magnetic skyrmions in metallic magnetic multilayers started only half a decade ago, but the progress achieved thus far has been tremendous, from stabilization of skyrmions at room temperature to controlled nucleation and motion. While magnetic random access memories might well replace the usual SRAM and DRAM in a few years from now, skyrmion-based race-tracks, thanks to their three-dimensional architecture potential could provide the replacement for hard disk drives or flash memories for high density data storage. This would be the second spintronics technical breakthrough after the discovery of the giant magneto-resistance. In this article, we reviewed our recent efforts to progress towards this goal, but also to unravel the fundamental properties of skyrmions and the associated Dzyaloshinskii-Moriya interaction.

\section{ACKNOWLEDGMENTS}

The authors acknowledge financial support from European Union grant MAGicSky No. FET-Open-665095, and thank J. Sampaio for providing the illustration of Fig.1(a) and S. Rohart for discussions in relation to subsection 5.2. Part of this work was performed at the Synchrotron SOLEIL, Gif-sur-Yvette, France (proposals 20150410 and 20161351), and the Swiss Light Source, Paul Scherrer Insitut, Villigen, Switzerland; the PolLux endstation was financed by the German Minister fr Bildung und Forschung (BMBF) through contracts 05KS4WE1/6 and 05KS7WE1.

\section{REFERENCES}

[1] N. Nagaosa and Y. Tokura, "Topological properties and dynamics of magnetic skyrmions," Nat. Nano. 8, pp. 899-911, 2013.

[2] N. S. Kiselev, A. N. Bogdanov, R. Schäfer, and U. K. Rößler, "Topological properties and dynamics of magnetic skyrmions," J. Phys. D: Appl. Phys. 44, p. 392001, 2011.

[3] N. Romming, A. Kubetzka, C. Hanneken, K. von Bergmann, and R. Wiesendanger, "Field-dependent size and shape of single magnetic skyrmions," Phys. Rev. Lett. 114, p. 177203, May 2015.

[4] R. Wiesendanger, "Nanoscale magnetic skyrmions in metallic films and multilayers: a new twist for spintronics," Nat. Rev. Materials 1, p. 16044, 2016.

[5] A. Fert, N. Reyren, and V. Cros, "Magnetic skyrmions: advances in physics and potential applications," Nat. Rev. Materials 2, p. 17031, 2017.

[6] C. Moreau-Luchaire, C. Moutafis, N. Reyren, J. Sampaio, C. A. F. Vaz, N. Van Horne, K. Bouzehouane, K. Garcia, C. Deranlot, P. Warnicke, P. Wohlhüter, J.-M. George, M. Weigand, J. Raabe, V. Cros, and A. Fert, "Additive interfacial chiral interaction in multilayers for stabilization of small individual skyrmions at room temperature," Nat. Nano. 11, pp. 444-448, 2016.

[7] G. Chen, T. Ma, A. T. NDiaye, H. Kwon, C. Won, Y. Wu, and A. K. Schmid, "Tailoring the chirality of magnetic domain walls by interface engineering," Nat. Commun. 4, p. 2671, 2013. 
[8] A. Hrabec, N. A. Porter, A. Wells, M. J. Benitez, G. Burnell, S. McVitie, D. McGrouther, T. A. Moore, and C. H. Marrows, "Measuring and tailoring the Dzyaloshinskii-Moriya interaction in perpendicularly magnetized thin films," Phys. Rev. B 90, p. 020402, Jul 2014.

[9] S. McVitie et al., "Skyrmion Néel walls in multilayer thin films with perpendicular magnetic anisotropy." In preparation.

[10] D. Maccariello, W. Legrand, N. Reyren, K. Garcia, K. Bouzehouane, S. Collin, V. Cros, and A. Fert, "Electrical signature of individual magnetic skyrmions in multilayered systems." arXiv:1706.05809. Submitted.

[11] M. J. Benitez, A. Hrabec, A. P. Mihai, T. A. Moore, G. Burnell, D. McGrouther, C. H. Marrows, and S. McVitie, "Magnetic microscopy and topological stability of homochiral nel domain walls in a $\mathrm{Pt} / \mathrm{Co} / \mathrm{AlO}_{x}$ trilayer," Nat. Commun. 6, p. 8957, 2015.

[12] J.-Y. Chauleau et al., "X-ray resonant magnetic scattering as a rapid tool to reveal domain wall textures in magnetic films." In preparation.

[13] H. A. Dürr, E. Dudzik, S. S. Dhesi, J. B. Goedkoop, G. van der Laan, M. Belakhovsky, C. Mocuta, A. Marty, and Y. Samson, "Chiral magnetic domain structures in ultrathin FePd films," Science 284(5423), pp. 2166$2168,1999$.

[14] W. Legrand, D. Maccariello, N. Reyren, K. Garcia, C. Moutafis, C. Moreau-Luchaire, S. Collin, K. Bouzehouane, V. Cros, and A. Fert, "Room-temperature current-induced generation and motion of sub-100 nm skyrmions," Nano Letters 17(4), pp. 2703-2712, 2017.

[15] W. Jiang, P. Upadhyaya, W. Zhang, G. Yu, M. B. Jungfleisch, F. Y. Fradin, J. E. Pearson, Y. Tserkovnyak, K. L. Wang, O. Heinonen, S. G. E. te Velthuis, and A. Hoffmann, "Blowing magnetic skyrmion bubbles," Science 349(6245), pp. 283-286, 2015.

[16] J. Iwasaki, M. Mochizuki, and N. Nagaosa, "Current-induced skyrmion dynamics in constricted geometries," Nat. Nano. 8, pp. 742-747, 2013.

[17] J. Sampaio, V. Cros, S. Rohart, A. Thiaville, and A. Fert, "Nucleation, stability and current-induced motion of isolated magnetic skyrmions in nanostructures," Nat. Nano. 8, p. 839844, 2013.

[18] A. Neubauer, C. Pfleiderer, B. Binz, A. Rosch, R. Ritz, P. G. Niklowitz, and P. Böni, "Topological hall effect in the A phase of MnSi," Phys. Rev. Lett. 102, p. 186602, May 2009.

[19] K. Zeissler, K. Shahbazi, J. Massey, S. Finizio, J. Raabe, F. A. Ma'Mari, M. C. Rosamond, E. H. Linfield, T. A. Moore, G. Burnell, and C. H. Marrows, "Direct imaging and electrical detection room temperature of a single skyrmion." arXiv:1706.06024.

[20] W. Jiang, X. Zhang, G. Yu, W. Zhang, X. Wang, M. Benjamin Jungfleisch, J. E. Pearson, X. Cheng, O. Heinonen, K. L. Wang, Y. Zhou, A. Hoffmann, and S. G. E. te Velthuis, "Direct observation of the skyrmion hall effect," Nat. Phys. 13, pp. 162-169, 2017.

[21] A. Vansteenkiste, J. Leliaert, M. Dvornik, M. Helsen, F. Garcia-Sanchez, and B. V. Waeyenberge, "The design and verification of MuMax3," AIP Advances 4(10), p. 107133, 2014.

[22] J.-V. Kim and M.-W. Yoo, "Current-driven skyrmion dynamics in disordered films," Applied Physics Letters 110(13), p. 132404, 2017.

[23] C. Reichhardt and C. J. O. Reichhardt, "Noise fluctuations and drive dependence of the skyrmion hall effect in disordered systems," New Journal of Physics 18(9), p. 095005, 2016. 